\title{
Morphological and Anatomical Studies on Some Taxa of Sub Family Malvoideae (Malvaceae s.l)
}

\author{
Wafaa M. Said ${ }^{\text {a }}$, Thoria R.Mohamed ${ }^{\text {a }}$, Abeer A. Elhalwagi ${ }^{\text {b }}$, and Zeinab M.Ahmed ${ }^{\mathrm{a}}$ \\ a Ain Shams University,Faculty of Womens for Arts,Science , and Education,Botany Department \\ ${ }^{\mathrm{b}}$ National Gene Bank ,Agriculture Research Center
}

\begin{abstract}
Morphological and anatomical studies were carried out on four ( two wild and two cultivated) species belonging to sub family Malvoideae (Malvaceae s.l) and collected from different regions in Egypt to assess the diagnostic value of these characters in taxa delimitation .The results obtained using UPGMA clustring method from analysis of 71 macro- $\&$ micro morphological characters resulting in a dendogram which indicated a close taxonomic relationship between Hibiscus esculentus and Hibiscus sabdariffa more than Malva parviflora with similarity index (76\% and $75 \%$ respectively). Dissimilarity was recorded in Sida spinosa from the other three species, it distinguished by high genetic diversity based on variation in morphological and anatomical features. The studied species were analyzed for assessment of taxonomic interrelationship among them.
\end{abstract}

Key word : Malvoideae ,Morphology , Anatomy ,Numerical analysis

\section{Introduction}

Malvoideae is a subfamily belongs to Malvaceae s.l (Alverson et al. 1999;Bayer et al.1999 ; Whitlock et al. 2001), with Bombacoideae constitute a well supported clade named Malvatheca (Baum et al. 1998). Malvoideae includes the traditional Malvaceae sensu strictu (Mallows or Eumalvoideae ) that comprises 78 genera and 1700 species (Baum et al. 2004). Malvaceae was divided into five tribes Abutileae, Decaschisteae, Hibisceae, Malvaceae and Ureneae(Paul and Naya , 1988) while it composed of nine tribes Abutileae, Decaschistieae, Gossypieae, Hibisceae, Kydieae, Malopeae, Malva visceae, Malveae, and Ureneae (Hinsley2006 ).

( Judd and Manchester 1997; Alverson et al. 1999;Bayer et al.1999 ) indicated that Malvaceae s.s is the only family in core families Malvales represents a monophyletic group. putatively early-branching Malvoideae have a predominantly Australasian/Asian distribution (Pfeil et al .,2002).Plants of subfamily Malvoideae are herbs, shrubs or trees ,erect or decumbent with stellate hairs and mucilage substance . Leaves simple, lobed or divided, Flowers solitary or in cymes, epicalyx useful characters to differentiated between genera. Five sepals and petals. five -many stamens, filaments form monoadelphous, epipetalous staminal tube, anther one cell . Fruit a loculicidal dehiscent or indehiscent capsule or schizocarp, rarely a berry. Seeds reniform or ovoid (Watson and Dallwitz1992 ) with high economical value; stems fibers of Hibicuse sabdriffa and sida used for making cordage, ropes, sacks and papers. roots of Althaea 
rosea and A. officinalis used in medicine. Abelmoschus esculents; fruits are vegetable and its stalks used as fibers . Gossypium arboreum, G. hirsutum; (fibers from the seeds are used in manufacture of textile, rubber- tyre fabrics ). Petals of Hibiscus rosa sinensis are used for caugh and coloring. Hibiscus sabdriffa : red fleshy epicalyx is edible. Many species of Malva are edible as leaf vegetables and drugs. sida species ; some are barely edible, and some as herbal medicine (Pandy 2006)

The objective of this work is to determine the taxonomic relationships among the four studied species of subfamily Malvoideae using morphological and anatomical characteristics .

\section{Material and Methods}

\section{Samples collection}

The four plant specimens for the present study were collected from different locations in Egypt, during 2014 and 2015 seasons. Plants were identified following standard literatures (Boulos,2000),(Täckholm,1974). (Table 1) shows the list of studied taxa and place of collection.

\section{Table (1): List of Studied Taxa and their locations}

\begin{tabular}{|l|l|l|}
\hline Sl.No. & Plant Name & Location \\
\hline 1 & *Hibiscus esculentus L. =(Ablemoscus esculentus) & El Monofia Governorate \\
2 & *Hibiscus sabdariffa L. & El Dakhlia Governorate \\
3 & Malva parviflora L. & El Dakhlia Governorate \\
4 & Sida spinosa L. & El Dakhlia Governorate \\
\hline
\end{tabular}

*cultivated species

\section{Morphological and Anatomical studies}

The macromorphology of four studied taxa including whole plant habit, stem, petiol, leaf ,flower and fruit was described from fresh specimens . the average length and width for different parts were taken in $\mathrm{cm}$. For micromorphology of stems and leaves, cross microtome sections of $10-20 \mu \mathrm{m}$ were cut and stained with safranin and light green according to method described by (Johansen , 1940). Photographed using LEICA dm LS2 microscope fixed with ICC50Hd camera . The anatomical descriptions were reviewed with (Metcalf and Chalk, 1979).

\section{Data analysis}

The presence or absence of the 90 macro-\&micromorphological characters were given the numerical code 0 ( absence) or 1(present) ( Table 2 ) .the similarity matrix was determined using PAST software (Hampl et al.,2001 )and phylogenetic dendogram was obtained from it following the Unweighted Pair Group Method using Arithmetic Averages (UPGMA ;Sneath and Sokal,1973) 


\section{Results and Discussion}

\section{Macro and micro morphological analysis}

The morphological and anatomical featurs were shown in Tables (2\&3), plates (1 -4). Morphologically ; the four studied species have similar characters such as presence of hairs, mucilage cells, alternate leaves, inflorescence type (cymes), pedicelate ,bisexual flowers ,five gamo sepals ,five poly petals numerous and epipetalous staminal tube, dorsifixed, monothecate anthers and lobed stigma. Anatomically ; they have crystals ,glandular hairs, cortical collenchyma, chlorenchyma ,mucilaginous cells in cortex and pith, vascular bundle arranged in one ring, wide pith and dorsiventral leaf blade.

The cluster analysis of 71 macro and micro morphological features produce a dendogram (Fig.1) illustrating that the four species of malvoideae divided into two clusters at taxonomic level (0.54) ; cluster I contain Sida spinosa, it specialized than others by circular stem, simple leaves with rounded base and serrate margin, short petiole, sepals shape and absence of epicalyx - Anatomically ;stem and leaf have high density and different types of hairs (glandular multicellular, shaggy ,stellate and unicellur filiform) ,two types of crystals (druses and solitary), stem with narrow inter vascular rays and triangular phloem medullary rays, adaxial surface of leaf was leveled, the previous observations agreed with that of the foliar trichomes however possessed a remarkable diversity and much variation is noticed in anatomical characters of stems of Sida (Arul \& Ida ,2017) and (Shaheen et al.,2009). They concluded that foliar epidermal features with particular emphasis on better terminology, macro-, micromorphology and distribution of different glandular and eglandular trichomes in Sida may be a useful taxonomic tool . Cluster II compises the other three species .

Hibiscus esculentus and H. sabdariffa were closely related to each other than Malva parviflora, at level (0.75) Malva parviflora separated in group I because it differs from them in its prosrate stem ,leaf lobes number, apex , base and shape ,carples number (9-10), discoid fruit , high density of hairs, thick cutical layer, that in the same line with the results of morphological and microscopical studies of stem, leaf and root of $M$. parviflora which may be helpful in the identification of these parts of this species (Akbar et al.,2014).

Separation between $H$.esculentus and $H$. sabdariffa in group II occurred at level 0.76) ,they similar in most characters as they Short lived perennial shrubs, pulvinus petiole, pedicel length, sepals shape and capsule fruit ,but they different in color of stem ,petiole, flower ,leaf shape, venation, leaf lobe type and stigma number. Anatomically $H$.esculentus had solitary crystals in the leaf , unicellular filiform trichomes ,radial ad axial cells, vascular bundles appeared in arc shape and stem phloem dissected. Distribution and quality of the crystals on the adaxial and abaxial epidermal surfaces of the two species $H$. sabdariffa and A. esculentus. , the differences in trichomes observed could used to separate the two species (Olotuah 2014 ). Table (4) represented the similarity index among the four species; the highest ratio between $H$ .esculentus and H. sabdariffa (76\%) and the lowest ratio between $H$.esculentus and S.spinosa (49\%). 
Table (2): Morphological characters and character states of the studied species

\begin{tabular}{|c|c|c|c|c|c|c|}
\hline No. & $\begin{array}{c}\text { Characters } \\
\text { species }\end{array}$ & state & 1 & 2 & 3 & 4 \\
\hline \multirow[t]{2}{*}{1} & \multirow[t]{2}{*}{ Habitat } & Cultivated & 1 & 1 & 0 & 0 \\
\hline & & wild & 0 & 0 & 1 & 1 \\
\hline \multirow[t]{2}{*}{2} & \multirow[t]{2}{*}{ Habit } & $\begin{array}{l}\text { Shortlived perennial } \\
\text { shrub }\end{array}$ & 1 & 1 & 0 & 0 \\
\hline & & Annual herb & 0 & 0 & 1 & 1 \\
\hline \multirow[t]{2}{*}{3} & \multirow[t]{2}{*}{ Stem type } & woody & 1 & 1 & $\overline{0}$ & 0 \\
\hline & & herbaceous & 0 & 0 & 1 & 1 \\
\hline \multirow[t]{2}{*}{4} & \multirow[t]{2}{*}{ Stem nature } & erect & 1 & 1 & $\overline{0}$ & 1 \\
\hline & & prostrate & 0 & 0 & 1 & 0 \\
\hline 5 & Stem texture & hairy & 1 & 1 & 1 & 1 \\
\hline \multirow[t]{2}{*}{6} & \multirow[t]{2}{*}{ Stem color } & green & 1 & 0 & 1 & 1 \\
\hline & & red & 0 & 1 & 0 & 0 \\
\hline \multirow[t]{2}{*}{7} & \multirow[t]{2}{*}{ Stem cross section } & Irregular Circle & 0 & 0 & 1 & 0 \\
\hline & & circular & 1 & 1 & $\overline{0}$ & 1 \\
\hline 8 & polymorphism & present & 1 & 1 & 0 & 0 \\
\hline \multirow[t]{2}{*}{9} & \multirow[t]{2}{*}{ Petiole base } & pulvinus & 1 & 1 & 0 & 0 \\
\hline & & normal & 0 & 0 & 1 & 1 \\
\hline \multirow[t]{2}{*}{10} & \multirow[t]{2}{*}{ Petiole texture } & Glabrous & 0 & 1 & 0 & 0 \\
\hline & & hairy & 1 & 0 & 1 & 1 \\
\hline \multirow[t]{2}{*}{11} & \multirow[t]{2}{*}{ Petiole color } & green & 1 & 0 & 1 & 1 \\
\hline & & red & 0 & 1 & 0 & 0 \\
\hline \multirow[t]{2}{*}{12} & \multirow[t]{2}{*}{ Petiole length } & Long $(5-12 \mathrm{~cm})$ & 1 & 1 & 1 & 0 \\
\hline & & Short ( less than $5 \mathrm{~cm}$ ) & 0 & 0 & 0 & 1 \\
\hline \multirow[t]{3}{*}{13} & \multirow[t]{3}{*}{ Stipules shape } & Tri angular & 1 & 0 & 0 & 0 \\
\hline & & lanceolate & 0 & 1 & 1 & 0 \\
\hline & & linear & 0 & 0 & 0 & 1 \\
\hline \multirow[t]{2}{*}{14} & \multirow[t]{2}{*}{ Leaf composition } & simple & 0 & 1 & 0 & 1 \\
\hline & & lobed & 1 & 1 & 1 & 0 \\
\hline \multirow[t]{3}{*}{15} & \multirow[t]{3}{*}{ Ieaf shape (outline) } & reniform & 0 & 1 & 1 & 0 \\
\hline & & Cordate & 1 & 0 & 0 & 0 \\
\hline & & Ovate & 0 & 0 & 0 & 1 \\
\hline 16 & lobe type & Palmatelyfid & 1 & 0 & 1 & 0 \\
\hline & & Palmatelysect & 0 & 1 & 0 & 0 \\
\hline 17 & lobe no & $3-5$ & 1 & 1 & 0 & 0 \\
\hline & & $5-7$ & 0 & 0 & 1 & 0 \\
\hline 18 & & Lanceolate & 0 & 1 & 0 & 0 \\
\hline & Lobe shape & Ovate & 1 & 0 & 0 & 0 \\
\hline & & Reniform & 0 & 0 & 1 & 0 \\
\hline 19 & Leaf /lobe color & Green & 1 & 0 & 1 & 1 \\
\hline
\end{tabular}


J. Sci. Res. Sci.,Vol.(35), 2018

\begin{tabular}{|c|c|c|c|c|c|c|}
\hline & & Green with red veins & 0 & 1 & 0 & 0 \\
\hline \multirow[t]{3}{*}{20} & \multirow[t]{3}{*}{ Leaf /lobe margin } & Serrate & 0 & 0 & 0 & 1 \\
\hline & & crenate & 1 & 0 & 1 & 0 \\
\hline & & Serrate-dentate & 0 & 1 & 0 & 0 \\
\hline \multirow[t]{3}{*}{21} & \multirow[t]{3}{*}{ Leaf /lobe apex } & acute & 0 & 1 & 0 & 1 \\
\hline & & mucronate & 1 & 0 & 0 & 0 \\
\hline & & obtuse & 0 & 0 & 1 & 0 \\
\hline \multirow[t]{4}{*}{22} & \multirow[t]{4}{*}{ Leaf base } & Truncate & 0 & 1 & 0 & 0 \\
\hline & & cordate & 0 & 0 & 1 & 0 \\
\hline & & acute & 1 & 0 & 0 & 0 \\
\hline & & rounded & 0 & 0 & 0 & 1 \\
\hline
\end{tabular}

\section{Continue table (2):}

\begin{tabular}{|c|c|c|c|c|c|c|}
\hline No. & $\begin{array}{r}\text { Characters } \\
\text { species }\end{array}$ & state & 1 & 2 & 3 & 4 \\
\hline \multirow[t]{2}{*}{23} & \multirow[t]{2}{*}{ Leaf size } & Large $8-10 \mathrm{~cm}$ & 1 & 1 & 1 & 0 \\
\hline & & Small $3 \mathrm{~cm}$ & 0 & 0 & 0 & 1 \\
\hline \multirow[t]{3}{*}{24} & \multirow[t]{3}{*}{ Leaf /lobe venation } & Reticulate & 1 & 1 & 1 & 1 \\
\hline & & Pinnatly unicostate & 0 & 1 & 0 & 1 \\
\hline & & palmatly multiicostate & 1 & 0 & 1 & 0 \\
\hline 25 & Inflorescence type & Cymes & 1 & 1 & 1 & 1 \\
\hline \multirow[t]{2}{*}{26} & \multirow[t]{2}{*}{ Flower Position } & axillary & 1 & 0 & 1 & 0 \\
\hline & & terminal & 0 & 1 & 0 & 1 \\
\hline \multirow[t]{4}{*}{27} & \multirow[t]{4}{*}{ Flower Color } & white & 0 & 0 & 1 & 0 \\
\hline & & pink & 0 & 1 & 0 & 0 \\
\hline & & Yellow- red color & 1 & 0 & 0 & 0 \\
\hline & & White -creamy & 0 & 0 & 0 & 1 \\
\hline \multirow[t]{2}{*}{28} & \multirow[t]{2}{*}{ Flower Size } & Moderate $2-5 \times 1-4 \mathrm{~cm}$ & 1 & 1 & 0 & 0 \\
\hline & & Small 0.5x.0.2 cm & 0 & 0 & 1 & 1 \\
\hline 29 & Epicalyxes . & present & 1 & 1 & 1 & 0 \\
\hline \multirow[t]{2}{*}{30} & \multirow[t]{2}{*}{ Pedicel length } & short & 1 & 1 & 0 & 0 \\
\hline & & v. short & 0 & 0 & 1 & 1 \\
\hline \multirow[t]{3}{*}{31} & \multirow[t]{3}{*}{ Sepals shape } & triangular & 0 & 0 & 0 & 1 \\
\hline & & ovate & 1 & 1 & 1 & 0 \\
\hline & & linear & 0 & 0 & 0 & 0 \\
\hline \multirow[t]{2}{*}{32} & \multirow[t]{2}{*}{ Texture } & glabrous & 0 & 1 & 1 & 0 \\
\hline & & Hairy & 1 & 0 & 0 & 1 \\
\hline \multirow[t]{2}{*}{33} & \multirow[t]{2}{*}{ Color } & green & 1 & 0 & 1 & 1 \\
\hline & & Red & 0 & 1 & 0 & 0 \\
\hline 34 & Petals shape & obovate & 1 & 1 & 1 & 1 \\
\hline 35 & Texture & glabrous & 1 & 1 & 1 & 1 \\
\hline 36 & color & white & 0 & 0 & 1 & 0 \\
\hline
\end{tabular}


J. Sci. Res. Sci.,Vol.(35), 2018

\begin{tabular}{|c|c|c|c|c|c|c|}
\hline & & pink & 0 & 1 & 0 & 0 \\
\hline & & Yellow with red color & 1 & $\overline{0}$ & 0 & 0 \\
\hline 37 & Stamens & $\begin{array}{l}\alpha, \text { epipetalous and forming } \\
\text { staminal tub }\end{array}$ & 1 & 1 & 1 & 1 \\
\hline 38 & Carpels no \&fusion & 5 & 0 & 1 & 0 & 1 \\
\hline & & $5-7$ & 1 & 0 & 0 & 0 \\
\hline & & $9-10$ & 0 & 0 & 1 & 0 \\
\hline 39 & Ovules no per locule & 1 & 0 & 0 & 1 & 1 \\
\hline & & many & 1 & 1 & 0 & 0 \\
\hline 40 & Stigma no & 5 & 0 & 1 & 1 & 1 \\
\hline & & $5-7$ & 1 & 0 & 0 & 0 \\
\hline 41 & Fruit type & capsule & 1 & 1 & 0 & 0 \\
\hline & & schizocarp & 0 & 0 & 1 & 1 \\
\hline 42 & Shape & ovoid & 1 & 1 & 0 & 1 \\
\hline & & disciod & 0 & 0 & 1 & 0 \\
\hline 43 & size & Moderate $2-5 \times 1-2.5 \mathrm{~cm}$ & 1 & 1 & 0 & 0 \\
\hline & & Small $0.2-0.4 \times 0.3-0.7 \mathrm{~cm}$ & 0 & 0 & 1 & 1 \\
\hline 44 & Seeds shape & reniform & 1 & 1 & 1 & 0 \\
\hline & & ovoid & 0 & 0 & 0 & 1 \\
\hline 45 & Color & brown & 1 & 0 & 1 & 1 \\
\hline & & black & 0 & 1 & 0 & 0 \\
\hline 46 & Size & Small $0.5 \times 0.3 \mathrm{~cm}$ & 1 & 1 & 0 & 0 \\
\hline & & v. small $0.1 \times 0.1 \mathrm{~cm}$ & 0 & 0 & 1 & 1 \\
\hline
\end{tabular}

Table (3): Anatomical characters and character state of the four studied species

\begin{tabular}{|c|c|c|c|c|c|c|}
\hline NO. & Characters & state & 1 & 2 & 3 & 4 \\
\hline \multirow[t]{2}{*}{1} & \multirow[t]{2}{*}{ Leaf adaxial shape } & ridged & 1 & 1 & 1 & 0 \\
\hline & & leveled & 0 & 0 & 0 & 1 \\
\hline \multirow[t]{2}{*}{2} & \multirow[t]{2}{*}{ Cuticle layer } & thin & 1 & 1 & 0 & 1 \\
\hline & & thick & 0 & 0 & 1 & 0 \\
\hline \multirow[t]{2}{*}{3} & \multirow{2}{*}{$\begin{array}{l}\text { Adaxial epidermal } \\
\text { cell }\end{array}$} & radial & 1 & 0 & 1 & 0 \\
\hline & & Tangential & 0 & 1 & 0 & 1 \\
\hline 4 & Hairs & present & 1 & 1 & 1 & 1 \\
\hline \multirow[t]{4}{*}{5} & \multirow[t]{4}{*}{ Hairs type } & stellate & 0 & 0 & 1 & 1 \\
\hline & & Glandular m.c & 1 & 1 & 1 & 1 \\
\hline & & unicellar filiform & 1 & 0 & 1 & 1 \\
\hline & & shaggy & 0 & 0 & 0 & 1 \\
\hline \multirow[t]{2}{*}{6} & \multirow[t]{2}{*}{ Hairs density } & Many & 0 & 0 & 1 & 1 \\
\hline & & Few & 1 & 1 & 0 & 0 \\
\hline 7 & Mesophyll type & dorsiventral & 1 & 1 & 1 & 1 \\
\hline 8 & Secretory cells & Mucilaginous cells & 1 & 1 & 1 & 1 \\
\hline
\end{tabular}


J. Sci. Res. Sci., Vol.(35), 2018

\begin{tabular}{|c|c|c|c|c|c|c|}
\hline \multirow[t]{2}{*}{9} & \multirow[t]{2}{*}{ Mechanical tissues } & collenchyma & 1 & 1 & 1 & 1 \\
\hline & & parenchyma & 1 & 1 & 1 & 1 \\
\hline \multirow[t]{2}{*}{10} & \multirow{2}{*}{$\begin{array}{l}\text { Vascular bundle } \\
\text { shape }\end{array}$} & Arc shape & 1 & 0 & 0 & 0 \\
\hline & & Centralgroup & 0 & 1 & $\overline{1}$ & 1 \\
\hline \multirow[t]{2}{*}{11} & \multirow[t]{2}{*}{ Crystals type } & Druses & 0 & 1 & 1 & 0 \\
\hline & & solitary & 1 & 0 & 0 & 0 \\
\hline \multirow[t]{2}{*}{12} & \multirow[t]{2}{*}{ Outline of stem } & circular & 0 & 0 & 0 & 1 \\
\hline & & wavy & 1 & 1 & 1 & 0 \\
\hline \multirow[t]{2}{*}{13} & \multirow[t]{2}{*}{ Cuticle layer } & thick & 0 & 0 & 1 & 0 \\
\hline & & thin & 1 & 1 & 0 & 1 \\
\hline 14 & Epidermal cells shape & Tangential & 1 & 1 & 1 & 1 \\
\hline \multirow[t]{4}{*}{15} & \multirow[t]{4}{*}{ Hairs type } & Stellate & 0 & 1 & 1 & 1 \\
\hline & & Glandular m.c & 1 & 1 & 1 & 1 \\
\hline & & unicellar filiform & 0 & 1 & 1 & 1 \\
\hline & & shaggy & 0 & 0 & 0 & 1 \\
\hline \multirow[t]{2}{*}{16} & \multirow[t]{2}{*}{ Hairs density } & Many & 0 & 0 & 0 & 1 \\
\hline & & few & 1 & 1 & 1 & 0 \\
\hline 17 & Cortical cell type & $\begin{array}{l}\text { Parenchyma,Collenchymas, } \\
\text { Chlorenchyma }\end{array}$ & 1 & 1 & 1 & 1 \\
\hline \multirow[t]{2}{*}{18} & \multirow[t]{2}{*}{ Crystals type } & Druses & 1 & 1 & 1 & 1 \\
\hline & & Solitary & 0 & 0 & 0 & 1 \\
\hline \multirow[t]{2}{*}{19} & \multirow[t]{2}{*}{ Crystals density } & Many & 0 & 0 & 0 & 1 \\
\hline & & few & 1 & 1 & 1 & 0 \\
\hline 20 & Secretory cells & Mucilaginous cells & 1 & 1 & 1 & 1 \\
\hline \multirow[t]{2}{*}{21} & \multirow[t]{2}{*}{ Pericycle } & Continuous ring & 0 & 0 & 0 & 1 \\
\hline & & $\begin{array}{l}\text { Dissected ring of sclernchymatos } \\
\text { tissue }\end{array}$ & 1 & 1 & 1 & 0 \\
\hline \multirow[t]{2}{*}{22} & \multirow[t]{2}{*}{ Phloem } & Continuous & 0 & 1 & 0 & 1 \\
\hline & & Dissected & 1 & 0 & 1 & 0 \\
\hline \multirow[t]{2}{*}{23} & \multirow{2}{*}{$\begin{array}{l}\text { Phloem medullary } \\
\text { rays }\end{array}$} & Radial & 1 & 1 & 1 & 0 \\
\hline & & Tri angular & 0 & 0 & 0 & 1 \\
\hline \multirow[t]{2}{*}{24} & \multirow[t]{2}{*}{ Xylem vessels } & Complete ring & 0 & 0 & 0 & 1 \\
\hline & & Dissected ring & 1 & 1 & 1 & 0 \\
\hline \multirow[t]{2}{*}{25} & \multirow[t]{2}{*}{ Inter vascular rays } & wide & 1 & 1 & 1 & 0 \\
\hline & & narrow & 0 & 0 & 0 & 1 \\
\hline
\end{tabular}


(a)

(b)
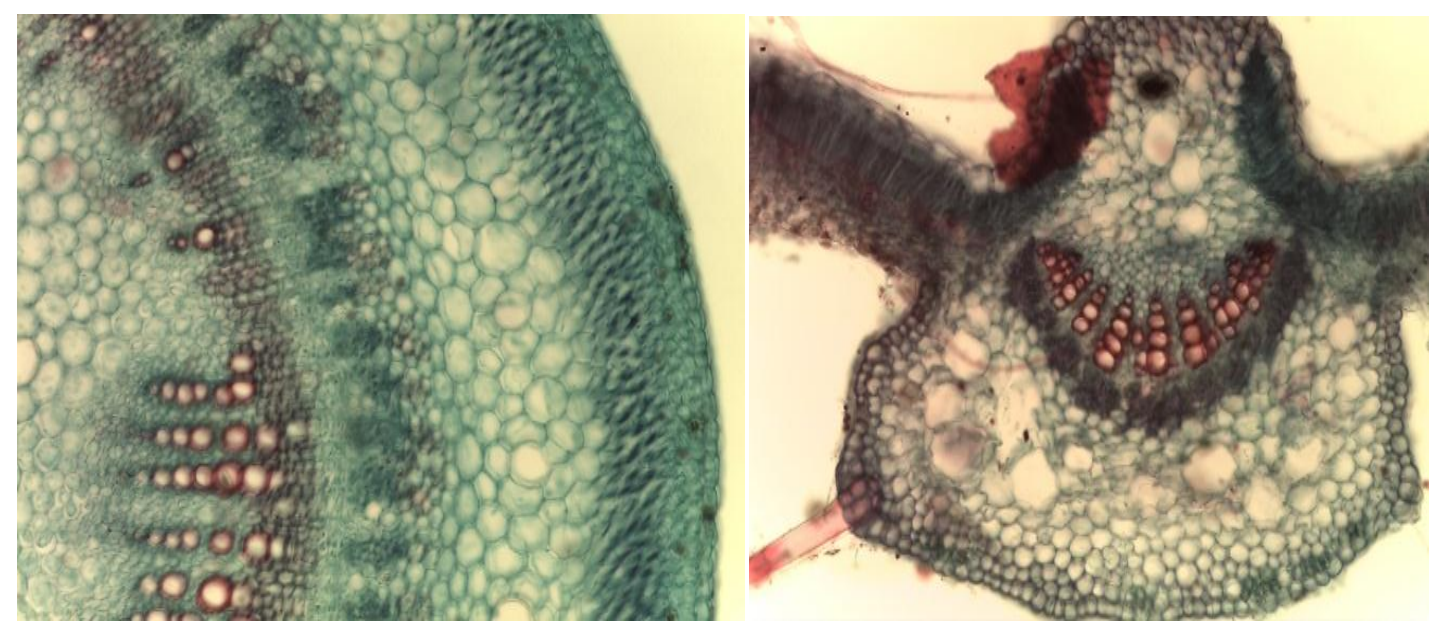

Plate (1): Anatomical characters of Hibiscus esculentus

(a)Cross section in stem (x40) (b) Cross section in leaf blade ( $x 40)$

(a)

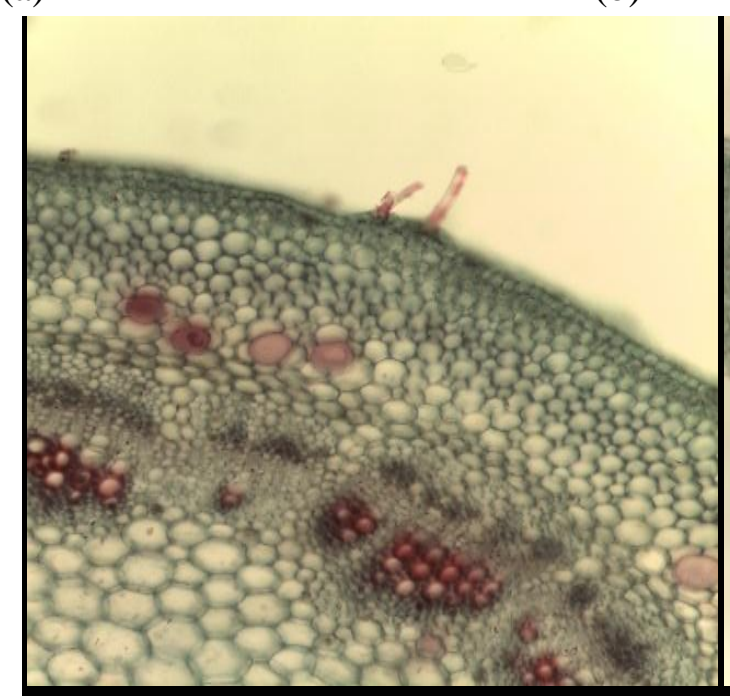

(b)

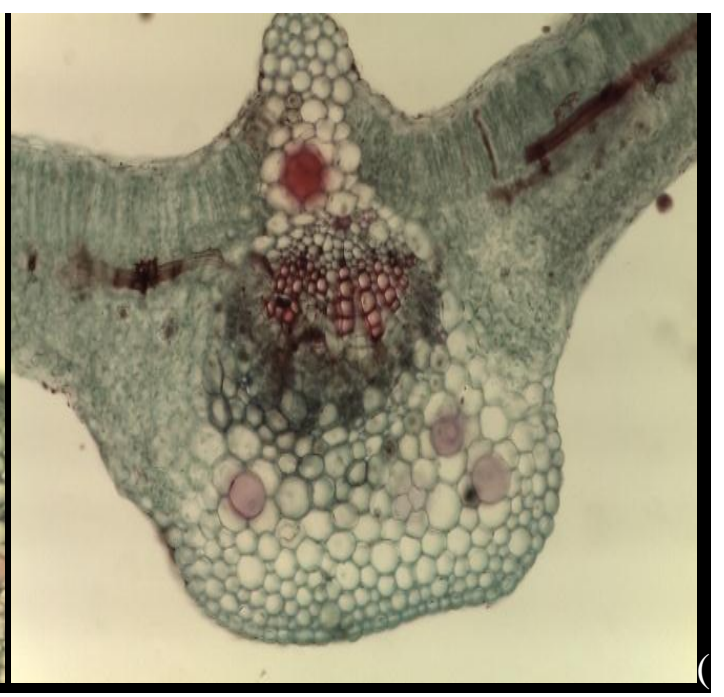

Plate (2): Anatomical characters of Hibiscus sabdariffa

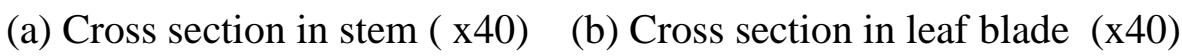


(a)

(b)
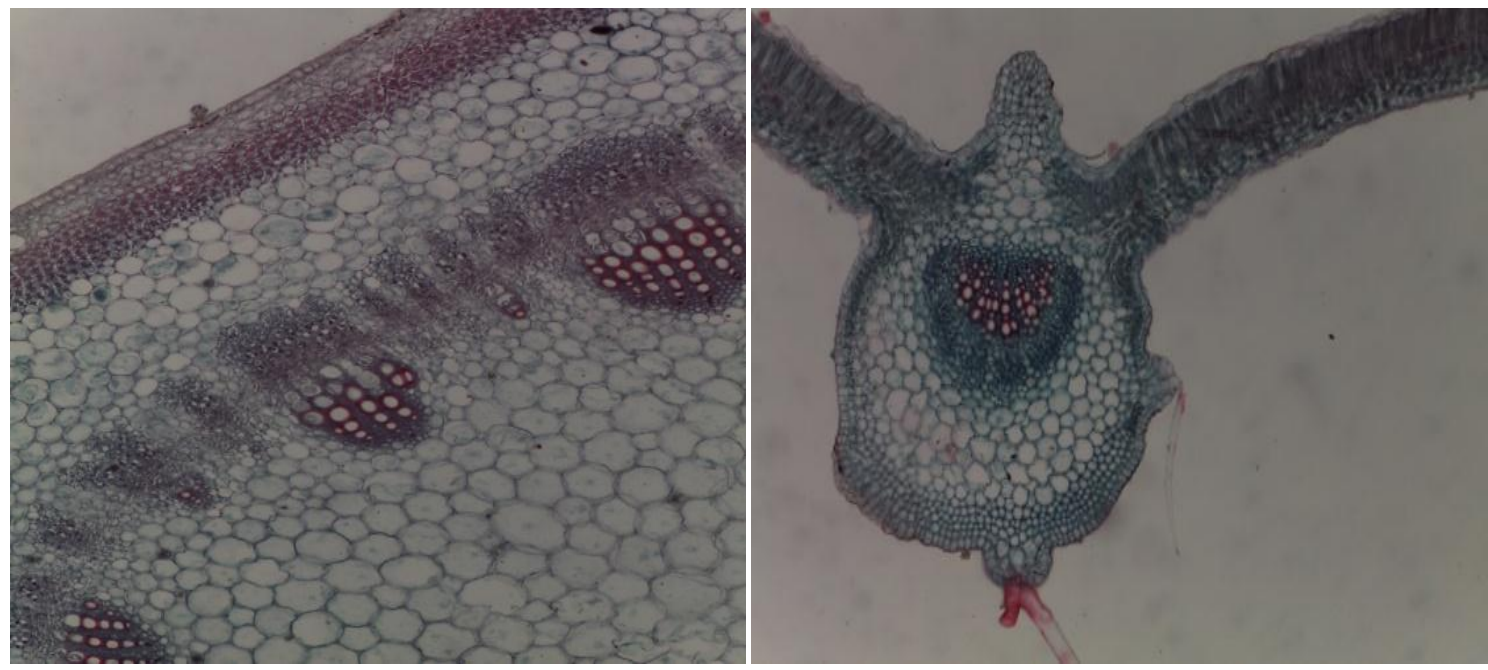

Plate (3): Anatomical characters of Malva parviflora

$\begin{array}{ll}\text { (a) Cross section in stem (x40) } & \text { (b) Cross section in leaf blade (x40) }\end{array}$

(a)

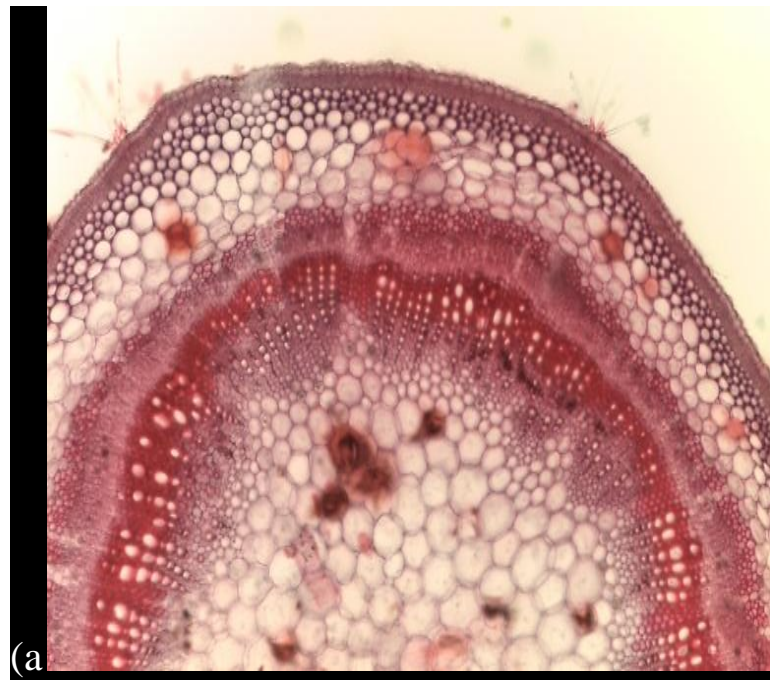

(b)

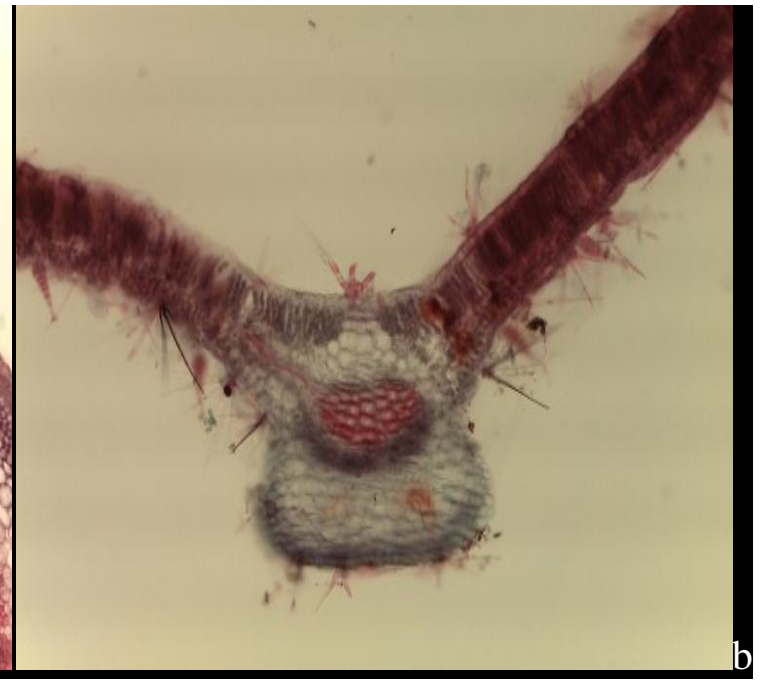

p

Plate (4) Anatomical characters of Sida spinosa $L$
(a) Cross section in stem ( $x 40)$
(b) Cross section in leaf blade ( $\mathrm{x} 40)$ 


\begin{tabular}{|c|c|c|c|c|c|c|c|}
\hline & & & & & & & \\
\hline 胥 & 용 & 品 & $=3$ & 承 & $\overrightarrow{\mathbf{z}}$ & 잉 & 8 \\
\hline
\end{tabular}

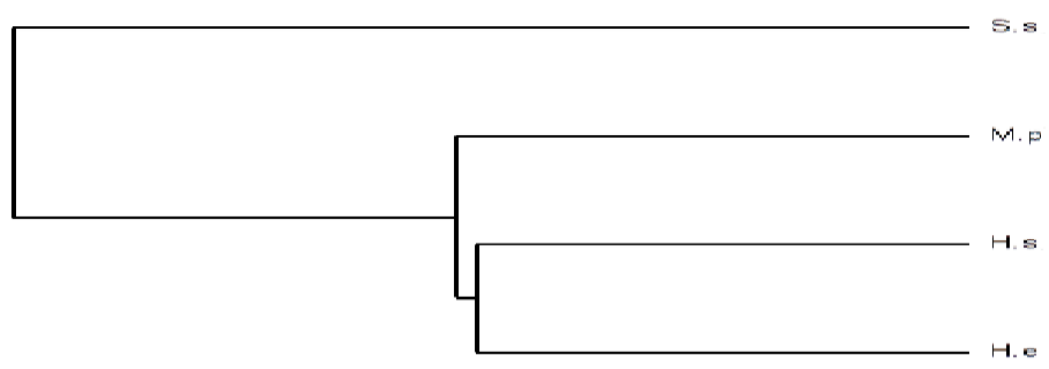

Fig.(1) :UPGMA -Dendogram based on 90 macro and micromorphological characters illustrating average taxonomic distance between four studied species .

Table (4) Similarity index of 4 species of subfamily Malvoideae using macro and micro morphological data

\begin{tabular}{|l|l|l|l|l|}
\hline & H.e1 & H.s2 & M.p3 & S.s4 \\
\hline H.e1 & 1 & & & \\
\hline H.s2 & 0.76 & 1 & & \\
\hline M.p3 & 0.75 & 0.75 & 1 & \\
\hline S.s4 & 0.49 & 0.58 & 0.52 & 1 \\
\hline
\end{tabular}




\section{CONCLUSION:}

There was a close relationship between Hibiscus esculentus and H. sabdariffa (cultivated plants) more than M.parviflora (wild plant). Sida spinosa (wild plant) which related to the same subfamily Malvoideae but it separated with single taxonomic distance from the other three species ; it has variation in many morphological and anatomical features .

\section{References:}

Akbar,S. , Hanif, U., Ali, J. and Ishtiaq, S. (2014) . Pharmacognostic studies of stem, roots and leaves of Malva parviflora L. Asian Pac Journal Trop Biomed.

Alverson, W.S., Whitlock, B.A., Nyffeler, R., Bayer, C. and Baum, D.A., (1999). Phylogenetic analysis of the core Malvales based on sequences of ndhF. American Journal of Botany.

Arul, A. A. A and Ida, J . C (2017) Morphological and Anatomical Variations Seen in Sida L., Kanyakumari District, Tamilnadu .International Journal of Scientific and Research Publications .

Baum, D.A., Alverson, W.S., and Nyffeler, R., (1998). A durian by any other name: taxonomy and nomenclature of the core Malvales. Harvard Pap. Botany. .

Baum, D.A., DeWitt Smith, S., Yen, A., Alverson, W.S., Nyffeler, R., Whitlock, B.A. and Oldham, R.L., (2004). Phylogenetic relationships of Malvatheca (Bombacoideae and Malvoideae; Malvaceae sensu lato) as inferred from plastid DNA sequences. American Journal of Botany.

Bayer, C., Fay, M.F., De Bruijn, A.Y., Savolainen, V., Morton, C.M., Kubitzki, K., Alverson, W.S., and Chase, M.W., (1999). Support for an expanded family concept of Malvaceae within a recircumscribed order Malvales: a combined analysis of plastid atpB and rbcL DNA sequences. Botany Journal. Linn. Soc.

Boulos L.( 2000) .Flora of Egypt. Vol. 2 . Cairo, Egypt: Al-Hadara Publishing .

Hampl,V.,Pavlicek ,A.and Flegr,J.(2001) .Construction and bootstrap analysis of DNAfingerprinting-based phylogenetic trees with the freeware program Free Tree: application to trichomonad parasites . International Journal Syst.Evol.Microbial .

Hinsley, S.R., (2006). Classification of Malvaceae: Overview, compostion: Position: Division.Malvaceae Info (Home). 
Johanson D.A(1940) Plant microtechnique ,Mc.Graw Hill Boot Company Inc.New York and London.

Judd, W.S., and Manchester, S.R., (1997). Circumscription of Malvaceae (Malvales) as determined by a preliminary cladistic analysis of morphological, anatomical, palynological, and chemical characters. Brittonia .

Metcalfe CR, Chalk L . (1979) Anatomy of the Dicotyledons Volume II Oxford University Press. Amen House ,London EC4.

Shaheen, N. , Khan, M. A. , Yasmin, G. , Ahmad, M., Mahmood T.,. Hayat, M.Q. and Zafar M., (2009) . African Journal of Biotechnology

Olotuah, O. F. (2014) .Anatomy of leaf epidermis and petiole of two selected species of Hibiscus Research Journal of Agriculture and Environmental Management.

Pandy B.P.(2006) . A text book of botany Angiosperms.S.Chand \&company LTD.Ramngar. Newdelhi.

Paul ,T. K and Naya, M. P., “Malvaceae” In: M. P. Nayar, K. Thothathri \& M. Sanjappa (1988) (eds.), Fascicles of Flora of India, Fascicle 19. Botanical Survey of India, Calcutta

Pfeil ,B.E. , Brubaker, C.L., Craven, L.A. and Crisp M.D.(2002). Phylogeny of Hibiscus and the tribe Hibisceae (Malvaceae) using chloroplast DNA sequences of ndhF and the rpl16 intron. Systematic Botany .

Sneath, P.H, Sokal R.(1973) Numerical Taxonomy, the principal and practice of Numerical classification. $2^{\text {nd }}$ Edn. Freeman (ED.) San Francisco, CA, USA.

Täckholm V. (1974). Students Flora of Egypt. $2^{\text {nd }}$ ed. Cairo University.

Watson, L. and Dalwitz, M.J., (1992). The Families of Flowering Plants: descriptions, illustrations, identification, and information .

Whitlock, B.A., Bayer, C. and Baum, D.A.,( 2001). Phylogenetic relationships and floral evolution of the Byttnerioideae ("Sterculiaceae" or Malvaceae s.1.) based on sequences of the chloroplast gene ndhF. Systematic of Botany. 


\section{الملخص باللغة العربية}

دراسة الثكل الظاهري والتركيب التشريحى لبعض الوحدات التصنيفية من تحت الفصيلة الخبازية ( للفصيلة الخبازية بالمعنى الواسع) الوحدات

زينب محروس أحمد' ، وفاء مرسى سعيد ' ، ثريا رشاد محمد '، عبير أحمد الحلوجى '

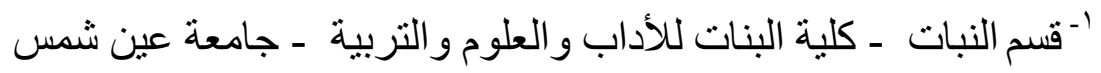

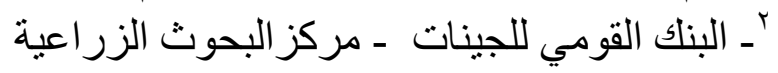

تمت دراسة ستة واربعون صفة مورفولوجية للنبات الكامل وخمسة وعشرون صفة تشريحية للساق

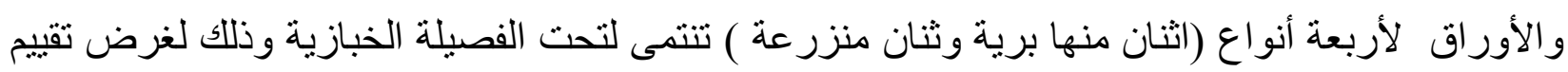
هذه الصفات لأستخدامها فى الفصل بين الأنواع محل الدراسة وقد جمعت من أماكن مختلفة فى مصر.

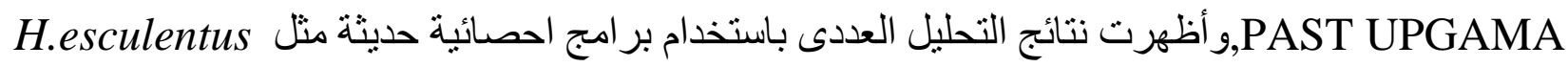
H.sabdarrifa

. أما نوع M.parviflora من حيث التشابة فى بعض الصفات المورفولوجية والتشريحية عن فقد تميز باختلاف فى الصفات المورفولوجية والتشريحية ولذلك ارتبط بعلاقة Sida spinosa تصنيفية ضعيفة مع بقية الأنواع المدروسة. 